Early mortality and survival improvements for adolescents and young adults with acute promyelocytic leukemia in California: an updated analysis

In the last two decades, survival from newly diagnosed acute promyelocytic leukemia (APL) has improved substantially after the introduction of all-trans retinoic acid (ATRA) and arsenic trioxide (ATO). ${ }^{1}$ Nevertheless, recent population-based studies revealed that mortality within 30 days of diagnosis remained high despite greater awareness of APL-specific coagulopathy and guidelines recommending prompt initiation of ATRA and aggressive supportive care as soon as APL is suspected. ${ }^{2,3}$ We previously showed that among patients aged $\leq 39$ years diagnosed with APL in California, 30-day mortality decreased from $26 \%$ before ATRA (1988-1995) to $14 \%$ after ATRA
(2004-2011). ${ }^{4}$ However, 7-day mortality did not differ between the pre- and post-ATRA eras. A higher risk of 30-day mortality and inferior overall survival were observed among patients without health insurance and those of Hispanic race/ethnicity. In this update, we examined whether early mortality decreased in the most recent eras of treatment and also examined overall survival trends among adolescents and young adults (15-39 years) with APL.

We used data from the California Cancer Registry linked to Medicaid enrollment files. Health insurance was classified based on Medicaid enrollment from 6 months prior to 6 months after APL diagnosis. The following categories were created: continuous Medicaid (enrollment 5 or 6 months prior to APL diagnosis), uninsurance or Medicaid at diagnosis (enrollment begins in the month prior to or within 2 months after diagnosis to account for reactive enrollment), discontinuous Medicaid

Table 1. Characteristics of adolescents and young adults $(n=524)$ with acute promyelocytic leukemia by period of diagnosis, California, 20052017.

\begin{tabular}{|c|c|c|c|c|}
\hline & $\begin{array}{c}\text { Pre-ACA }{ }^{\dagger} \\
(2005-2010) \\
N=216 \\
n(\%)\end{array}$ & $\begin{array}{l}\text { Period of diagnosis } \\
\text { Early ACA }{ }^{\ddagger} \\
\begin{array}{c}\text { (2010-2013) } \\
N=144 \\
n(\%)\end{array}\end{array}$ & $\begin{array}{c}\text { Full ACA }{ }^{\&} \\
\begin{array}{c}\text { (2014-2017) } \\
N=164 \\
n(\%)\end{array}\end{array}$ & P-valué \\
\hline \multicolumn{5}{|l|}{ Early mortality } \\
\hline 7-day mortality & $28(13.0)$ & $11(7.6)$ & $9.0(5.5)$ & 0.011 \\
\hline 30-day mortality & $31(14.4)$ & $15(10.4)$ & $12(7.3)$ & 0.029 \\
\hline Median age at diagnosis (IQR), years & $29(22-35)$ & $29(23-35)$ & $28(23-33)$ & 0.567 \\
\hline \multicolumn{5}{|l|}{ Sex } \\
\hline Female & $103(47.7)$ & $78(54.2)$ & $85(52.0)$ & 0.458 \\
\hline Male & $113(52.3)$ & $66(45.8)$ & $79(48.2)$ & \\
\hline \multicolumn{5}{|l|}{ Health insurance* } \\
\hline Private/military ${ }^{\&}$ & $94(43.5)$ & $59(41.0)$ & $49(29.9)$ & $<0.001$ \\
\hline Continuous Medicaid $^{*}$ & $30(13.9)$ & $20(13.9)$ & $66(40.2)$ & \\
\hline Discontinuous Medicaid" & $14(6.5)$ & $15(10.4)$ & $13(7.9)$ & \\
\hline Medicaid at diagnosis/uninsured ${ }^{\mu}$ & $61(28.2)$ & $47(32.6)$ & $30(18.3)$ & \\
\hline \multicolumn{5}{|l|}{ Care facility } \\
\hline NCI-CC & $77(35.7)$ & $54(37.5)$ & $68(41.5)$ & 0.507 \\
\hline Non-NCI-CC & $139(64.4)$ & $90(62.5)$ & $96(58.5)$ & \\
\hline \multicolumn{5}{|l|}{ Race/ethnicity** } \\
\hline Non-Hispanic White & $62(28.7)$ & $45(31.3)$ & 35 (21.3) & 0.472 \\
\hline Non-Hispanic Black & $12(5.6)$ & $6(4.2)$ & $14(8.5)$ & \\
\hline Hispanic & $113(52.3)$ & $76(52.8)$ & $88(53.7)$ & \\
\hline Asian/Pacific Islander & $25(11.6)$ & $16(11.1)$ & $24(14.6)$ & \\
\hline \multicolumn{5}{|l|}{ Neighborhood SES (tertiles) } \\
\hline Highest & $54(25.0)$ & $32(22.2)$ & $44(26.8)$ & 0.271 \\
\hline Medium & $72(33.3)$ & $62(43.1)$ & $52(31.7)$ & \\
\hline Lowest & $90(41.7)$ & $50(34.7)$ & $68(41.5)$ & \\
\hline Total deceased & 49 (22.7) & $19(13.2)$ & $12(7.3)$ & $<0.001$ \\
\hline
\end{tabular}

ACA: Affordable Care Act; SES: socioeconomic status; NCI-CC: National Cancer Institute-Designated Cancer Center; IQR: interquartile range. ${ }^{\dagger}$ Pre-ACA: March 2005 to September 2010; ${ }^{\ddagger}$ Early ACA: October 2010 to December 2013; \&Full ACA: 2014 to 2017. ${ }^{\&}$ Private insurance includes: Military, Health Maintenance Organization, Preferred Provider Organization, and Medicare. ${ }^{\ddagger}$ Continuous Medicaid: enrollment 5 or 6 months prior to diagnosis. ${ }^{\mu}$ Medicaid at diagnosis/uninsurance: coverage beginning in the month prior to or within 2 months after diagnosis to account for reactive enrollment. "Discontinuous Medicaid: enrollment that does not meet the definitions for continuous enrollees or Medicaid at diagnosis or Medicaid insurance recorded in the California Cancer Registry but without a match in the Medicaid enrollment files. * Other public $(n=3,0.6 \%)$ and unknown $(n=23,4.4 \%)$ insurances are not presented in the table. Other public insurance includes Indian/ Public Health Service, County Funded not otherwise specified (NOS); Medicare without supplement; Medicare, NOS. ** Other race/ethnicity (American Indian, $n=4)$ and unknown ( $\mathrm{n}=4)$ are not presented in the table. Data on Hispanics ${ }^{14}$ and Asian/Pacific Islanders ${ }^{15}$ are derived from a combination of variables based on the North American Association of Central Cancer Registries. $\mathrm{a} \chi^{2} P$-values were used to compare frequency distributions of sociodemographic and clinical characteristics by treatment.Tests for trends were used to examine early mortality by treatment era. 
Table 2. Relation of early mortality and overall survival with period of diagnosis, sociodemographic factors and care facility, California, 20052017.

$\begin{array}{cccc} & \text { 7-day mortality } & \text { 30-day mortality } & \text { Overall survival } \\ \text { N. of patients (\%) } & \text { Adjusted } \mathrm{OR}^{\S} & \text { Adjusted } \mathrm{OR}^{\S} & \text { Adjusted HR } \\ & (95 \% \mathrm{Cl}) & (95 \% \mathrm{Cl}) & (95 \% \mathrm{Cl})\end{array}$

\section{Period of diagnosis}

\begin{tabular}{|c|c|c|c|c|}
\hline Pre-ACA ${ }^{\dagger}$ & $216(41.2)$ & Reference & Reference & Reference \\
\hline Early ACA & $144(27.5)$ & $0.57(0.27-1.23)$ & $0.72(0.36-1.44)$ & $0.60(0.35-1.03)$ \\
\hline Full ACA ${ }^{\&}$ & $164(31.3)$ & $0.42(0.18-0.98)$ & $0.51(0.24-1.08)$ & $0.39(0.20-0.76)$ \\
\hline
\end{tabular}

\begin{tabular}{|c|c|c|c|c|}
\hline \multicolumn{5}{|l|}{ Sex } \\
\hline Female & $266(50.8)$ & Reference & Reference & Reference \\
\hline Male & $258(49.2)$ & $1.21(0.64-2.30)$ & $0.99(0.55-1.78)$ & $1.25(0.78-1.98)$ \\
\hline
\end{tabular}

Age at diagnosis (years)

\begin{tabular}{|c|c|c|c|c|}
\hline $15-20$ & $95(18.1)$ & Reference & Reference & Reference \\
\hline $21-25$ & $100(19.1)$ & $1.07(0.39-2.90)$ & $0.72(0.28-1.83)$ & $0.94(0.45-1.96)$ \\
\hline $26-39$ & $329(62.8)$ & $0.87(0.38-1.98)$ & $0.77(0.37-1.63)$ & $0.93(0.52-1.67)$ \\
\hline \multicolumn{5}{|l|}{ Race/ethnicity* } \\
\hline Non-Hispanic White & $142(27.1)$ & Reference & Reference & Reference \\
\hline Non-Hispanic Black & $32(6.1)$ & $0.95(0.18-4.99)$ & $1.19(0.28-4.98)$ & $1.27(0.45-3.64)$ \\
\hline Hispanic & $277(52.9)$ & $1.97(0.82-4.74)$ & $2.16(0.95-4.94)$ & $1.56(0.84-2.91)$ \\
\hline Asian/Pacific Islander & $65(12.4)$ & $1.45(0.43-4.92)$ & $1.25(0.38-4.10)$ & $1.40(0.60-3.23)$ \\
\hline \multicolumn{5}{|l|}{ Neighborhood SES } \\
\hline Highest & $130(24.8)$ & Reference & Reference & Reference \\
\hline Middle & $186(35.5)$ & $1.44(0.54-3.87)$ & $1.38(0.55-3.46)$ & $1.04(0.52-2.08)$ \\
\hline Lowest & $208(39.7)$ & $2.09(0.78-5.60)$ & $2.27(0.91-5.66)$ & $1.69(0.86-3.33)$ \\
\hline \multicolumn{5}{|l|}{ Care facility } \\
\hline NCI-CC & $199(38.0)$ & Reference & Reference & Reference \\
\hline Non-NCI-CC & $325(62.0)$ & $4.85(1.96-12.0)$ & $5.24(2.26-12.2)$ & $3.71(1.97-6.99)$ \\
\hline \multicolumn{5}{|l|}{ Health insurance* } \\
\hline Private/Military ${ }^{\&}$ & $202(38.6)$ & Reference & Reference & Reference \\
\hline Continuous Medicaid $^{*}$ & $116(22.1)$ & $0.77(0.29-2.07)$ & $0.71(0.29-1.76)$ & $0.86(0.42-1.77)$ \\
\hline Discontinuous Medicaid" & $42(8.0)$ & $1.12(0.35-3.57)$ & $1.28(0.46-3.61)$ & $1.52(0.70-3.29)$ \\
\hline Medicaid at diagnosis/uninsured ${ }^{\mu}$ & $138(26.3)$ & $1.00(0.44-2.29)$ & $0.98(0.46-2.10)$ & $1.04(0.57-1.92)$ \\
\hline
\end{tabular}

OR: odds ratio; HR: hazard ratio; ACA: Affordable Care Act; SES: socioeconomic status; NCI-CC, National Cancer Institute-Designated Cancer Center. ${ }^{\dagger}$ Pre-ACA: March 2005 to September 2010, ${ }^{\ddagger}$ Early ACA: October 2010 to December 2013; ${ }^{\&}$ Full ACA: 2014 to $2017{ }^{*}$ Other/unknown race/ethnicity and other/unknown health insurance was omitted from the table due to the small number of events. Data on Hispanics ${ }^{14}$ and Asian/Pacific Islanders ${ }^{15}$ are derived from a combination of variables based on the North American Association of Central Cancer Registries. ${ }^{\&}$ Private insurance includes: Military, Health Maintenance Organization, Preferred Provider Organization, and Medicare. ${ }^{\sharp}$ Continuous Medicaid: enrollment 5 or 6 months prior to the diagnosis of acute prolmyelocytic leukemia. ${ }^{\mu}$ Medicaid at diagnosis/uninsurance: coverage beginning in the month prior to or within 2 months after diagnosis to account for reactive enrollment. "Discontinuous Medicaid: enrollment that does not meet the definitions for continuous enrollees or Medicaid at diagnosis or Medicaid insurance recorded in the California Cancer Registry but without a match in the Medicaid enrollment files ${ }^{\S}$ Multivariable logistic regression (for early mortality) and Cox proportional regression models (for overall survival) were adjusted for all variables in the table.The proportional hazard assumption, assessed by examining log-log survival plots and confirmed using Schoenfeld residuals, was met for all variables in the multivariate Cox regression model.

(enrollment that does not meet the definitions for continuous enrollees, Medicaid at diagnosis or Medicaid insurance recorded in the California Cancer Registry but without a match in the Medicaid enrollment files), private, other public or unknown. We included three periods of diagnosis that reflect insurance policy changes in California under the Affordable Care Act (ACA): March 2005 to September 2010 (pre-ACA), October 2010 to December 2013 (early ACA: Dependent Care Expansion and early Medicaid expansion), and January 2014 to December 2017 (full ACA: Medicaid expansion and private health insurance marketplace). Neighborhood socioeconomic status is an aggregate measure at the census block level, ${ }^{5}$ which contains U.S. Census or American Community Survey information on education, occupation, unemployment, household income, poverty, rent and house values.
We examined the associations of 7- and 30-day mortality and overall survival with period of diagnosis, sociodemographic characteristics, and care facility using multivariable logistic regression and Cox proportional hazards regression, respectively. Results are presented as odds ratios (OR) or hazard ratios (HR) and corresponding $95 \%$ confidence intervals (95\% CI). We also estimated 3-year overall survival using the Kaplan-Meier method. Patients were followed from the date of diagnosis to the date of death, loss to follow-up or end of the study (December 31, 2018), whichever occurred first.

Five-hundred twenty-four adolescents and young adults diagnosed with APL between 2005 and 2017 were included in the analyses. Of these, $50.8 \%$ were female, $52.9 \%$ were of Hispanic race/ethnicity, $38.6 \%$ had private insurance, and $39.7 \%$ resided in the lowest socioeconomic neighborhoods. Fewer patients $(38.0 \%)$ were 
treated at National Cancer Institute-Designated Cancer Centers (NCI-CC). Overall, 7- and 30-day mortality were $9.2 \%$ and $11.1 \%$, respectively. From the pre-ACA to full ACA era, 7-day mortality decreased from $13.0 \%$ to $5.5 \%$ $(P$-value for trend $=0.011)$ and 30 -day mortality decreased from $14.4 \%$ to $7.3 \%$ ( $P$-value for trend $=0.029)$ (Table 1$)$. With a median follow-up of 4.7 years (range, $0-14.1), 80$ patients died during the study period: $48(60 \%)$ died within 7 days and $58(72.5 \%)$ died within 30 days of the diagnosis of APL. The 3-year survival increased from $79.8 \%$ before the ACA to $92.7 \%$ in the full ACA era $(P$ value for trend=0.0004).

Across treatment eras, there was a decrease in adolescents and young adults who were uninsured or obtained Medicaid insurance at APL diagnosis, from $28.2 \%$ (preACA) to $18.3 \%$ (full ACA). Among those, a few adolescents and young adults $(n=9,1.7 \%)$ remained uninsured in the pre- and early-ACA eras, but none was uninsured in the full ACA era. In addition, there was a substantial increase in continuous Medicaid enrollment prior to diagnosis, from $13.9 \%$ (pre-ACA) to $40.2 \%$ (full ACA) $(P$-value $<0.0001)$. Furthermore, we observed a trend towards increased initial care at NCI-CC, from 35.7\% before the ACA to $41.5 \%$ in the full ACA era, although this difference was not statistically significant.

In multivariable models, compared with the pre-ACA era, the odds of both 7- and 30-day mortality were about $50-60 \%$ lower in the full ACA era (OR=0.42, 95\% CI: $0.18-0.98$ and $\mathrm{OR}=0.51,95 \% \mathrm{CI}: 0.24-1.08$, respectively) (Table 2). Remarkably, the odds of 7- and 30-day mortality were approximately 5-fold higher among adolescents and young adults who did not receive initial care at a NCI-CC (OR=4.85, 95\% CI: $1.96-12.0$ and $\mathrm{OR}=5.28$, 95\% CI: 2.26-12.2, respectively). Likewise, the hazard of death decreased in the full ACA era compared to that in the pre-ACA era ( $\mathrm{HR}=0.39,95 \% \mathrm{CI}: 0.20-0.76)$, and was nearly 4 times higher for patients who did not receive initial care at a NCI-CC (HR=3.71, 95\% CI: 1.97-6.99). Age at diagnosis, sex, race/ethnicity, socioeconomic status and health insurance were not associated with early mortality or overall survival.

Our findings of reduced early mortality and improved overall survival during the study period are relevant. Most early deaths after APL result from severe hemorrhage (intracranial and, less often, pulmonary) and are the major cause of treatment failure. ${ }^{6}$ Another potentially fatal manifestation of APL is thrombosis (e.g., stroke, acute myocardial infarction, and pulmonary embolism). These manifestations can occur within a few hours or days of diagnosis or even prior to the recognition of APL, supporting the concept that APL should be considered as a medical emergency. ${ }^{7}$ Thus, timely access to optimal treatment is lifesaving. Several factors may have contributed to the better outcomes we observed among adolescents and young adults with APL in the more recent treatment eras. These include increased awareness of APL-related coagulopathy, improved physicians' adherence to treatment guidelines, and early referral to hematology/oncology centers. 6,8

In our previous study, Hispanic patients and those without health insurance experienced worse outcomes. In this update, we did not observe differences in early mortality or survival by race/ethnicity. This observation is encouraging and is likely the result of the increased insurance coverage we observed among Hispanics over time (uninsurance decreased from $46.3 \%$ before the ACA to $21.1 \%$ in the full ACA era). Historically, in the USA, adolescents and young adults have been the most highly uninsured or underinsured population. ${ }^{9}$ It is likely that adolescents and young adults without health insurance delay seeking medical care until the APL symptoms become severe, whereas those with insurance may be encouraged to seek medical attention earlier, when the first manifestations of APL appear. In this current study, we found increases in continuous Medicaid and reduction in Medicaid enrollments at diagnosis or uninsurance among adolescents and young adults with APL. Before implementation of the ACA, a restricted number of adults with a cancer diagnosis would qualify for Medicaid coverage. ${ }^{10}$ In California, a Medicaid expansion state, the ACA increased health insurance coverage to low-income young adults through the Dependent Coverage Expansion and Low-Income Health Plan as early as 2010. At the beginning of 2014, the Low-Income Health Plan ended, and patients obtained coverage under the California regular Medicaid program (Medi-Cal) or acquired private health insurance through the establishment of marketplace coverage. Marketplace insurance plans are prohibited to deny coverage to insurers or set higher premiums for pre-existing conditions such as cancer. ${ }^{10}$ Although we did not find an association between insurance and APL outcomes in our cohort, when we separately analyzed patients who remained uninsured $(n=9)$, we found that their 7-day and 30-day mortality were significantly higher. This suggests that uninsured patients are at risk of not having prompt access to optimal care during the initial diagnosis of APL.

Importantly, we found that higher early mortality and worse survival were strongly associated with location of initial care, consistent with prior reports. ${ }^{2}$ This may be related, in part, to greater awareness and provider expertise gained by treating a larger volume of patients with acute leukemias at NCI-CC. ${ }^{11}$ In addition, these facilities generally have better access to blood products and broader specialist support to care for severely ill patients. Changes in therapy during the study period are risk-stratified approaches that include the combination of ATRA and ATO, cytotoxic chemotherapy for high-risk patients, pre-emptive therapy for differentiation syndrome, and more aggressive blood product support for coagulopathy. ${ }^{8,12,13}$ Adherence to more modern approaches is more likely at NCI-CC and may have contributed to the improvement in outcomes we observed. In California, there are eight NCI-CC in seven cities, so timely access to NCI-CC may not be possible for many adolescents and young adults with APL, reinforcing the need to improve care at non-NCI-CC.

Our study limitations include a lack of treatment details, which prevented us from assessing adherence to treatment guidelines. In addition, we did not have clinical information for APL risk stratification. These data could contribute to our understanding of different factors that can influence early mortality in young patients with APL. Regardless of these limitations, we used populationbased data from the most populous and diverse U.S. state where cancer registration is mandatory, providing information on virtually all adolescents and young adults diagnosed with APL during the study period. We showed a considerable reduction in early mortality and increased survival over time. The higher 7-day and 30-day mortality and lower survival observed in adolescents and young adults cared for outside of NCI-CC underscore the need for earlier recognition and better implementation of treatment guidelines, particularly at non-NCI-CC.

Renata Abrahão, ${ }^{1,2}$ Raul C. Ribeiro, ${ }^{3}$ Marcio H. Malogolowkin, ${ }^{4}$ Ted Wun ${ }^{1}$ and Theresa H.M. Keegan ${ }^{1}$ 
${ }^{1}$ Center for Oncology Hematology Outcomes Research and Training (COHORT), Division of Hematology and Oncology, University of California Comprehensive Cancer Center, Sacramento, CA; ${ }^{2}$ Center for Healthcare Policy and Research, University of California, Davis, School of Medicine, Sacramento, CA; ${ }^{3}$ Department of Oncology, Division of Hematology and Oncology, St. Jude Children's Research Hospital, Memphis, TN and ${ }^{4}$ Department of Pediatrics, Division of Hematology and Oncology, University of California, Davis, School of Medicine, Sacramento, CA, USA

\section{Correspondence:}

RENATA ABRAHAO - rabrahao@ucdavis.edu

\section{doi:10.3324/haematol.2021.278851}

Received: March 26, 2021.

Accepted: May 4, 2021.

Pre-published: July 29, 2021.

Disclosures: no conflicts of interest to disclose.

Contributions: RA and THMK conceived and designed the study; THMK and TW acquired data; RA analyzed data and drafted the manuscript. All authors reviewed and approved the final version of the manuscript.

Funding: RA was supported by the Health Resources and Services Administration (HRSA) of the U.S. Department of Health and Human Services (HHS) under grant number T32HP30037 for Research in Primary Care. THMK was supported by the UC Davis Comprehensive Cancer Center (P30CA093373). TW was supported by UL1 0000860, National Center for Advancing Translational Science, National Institute of Health (NIH). RCR was partially funded by the National Cancer Institute (NCI) grant CA21765 and by the American Lebanese and Syrian Associated Charities. The collection of cancer incidence data used in this study was supported by the California Department of Public Health pursuant to California Health and Safety Code Section 103885; Centers for Disease Control and Prevention's (CDC) National Program of Cancer Registries, under cooperative agreement 5NU58DP006344; the NCI's Surveillance, Epidemiology and End Results (SEER) Program under contract HHSN261201800032I awarded to the University of California, San Francisco, contract HHSN261201800015I awarded to the University of Southern California, and contract HHSN261201800009I awarded to the Public Health Institute. The ideas and opinions expressed herein are those of the authors and do not necessarily reflect the opinions of the State of California, Department of Public Health, the NCI, and the $C D C$ or their Contractors and Subcontractors. Likewise, they should not be construed as the official position or policy of, nor should any endorsements be inferred by HRSA, HHS or the U.S. Government.

\section{References}

1. Sanz MA, Grimwade D, Tallman MS, et al. Management of acute promyelocytic leukemia: recommendations from an expert panel on behalf of the European LeukemiaNet. Blood. 2009;113(9):18751891

2. Ho G, Li Q, Brunson A, et al. Complications and early mortality in patients with acute promyelocytic leukemia treated in California. Am J Hematol. 2018;93(11): E370-E372.

3. Lehmann S, Deneberg S, Antunovic P, et al. Early death rates remain high in high-risk APL: update from the Swedish Acute Leukemia Registry 1997-2013. Leukemia. 2017;31(6):1457-1459.

4. Abrahao R, Ribeiro RC, Medeiros BC, et al. Disparities in early death and survival in children, adolescents, and young adults with acute promyelocytic leukemia in California. Cancer. 2015; 121(22):3990-3997.

5. Yost K, Perkins C, Cohen R, et al. Socioeconomic status and breast cancer incidence in California for different race/ethnic groups. Cancer Causes Control. 2001;12(8):703-711.

6. Abla O, Lo-Coco F, Sanz MA. Acute Promyelocytic Leukemia. First edition: Springer International Publishing (Chapter 6). 2018.

7. Lo-Coco F, Cicconi L, Voso MT. Progress and criticalities in the management of acute promyelocytic leukemia. Oncotarget. 2017; 8(59): 99221-99222.

8. Burnett AK, Russell NH, Hills RK, et al. Arsenic trioxide and all-trans retinoic acid treatment for acute promyelocytic leukaemia in all risk groups (AML17): results of a randomised, controlled, phase 3 trial. Lancet Oncol. 2015;16(13):1295-1305.

9. Kirchhoff AC, Lyles CR, Fluchel M, et al. Limitations in health care access and utilization among long-term survivors of adolescent and young adult cancer. Cancer. 2012;118(23):5964-5972.

10. Zhao J, Mao Z, Fedewa SA, et al. The Affordable Care Act and access to care across the cancer control continuum: a review at 10 years. CA Cancer J Clin. 2020;70(3):165-181.

11. Giri S, Pathak R, Aryal MR, et al. Impact of hospital volume on outcomes of patients undergoing chemotherapy for acute myeloid leukemia: a matched cohort study. Blood. 2015;125(21):3359-3360.

12. Lo-Coco F, Di Donato L, Schlenk RF. Targeted therapy alone for acute promyelocytic leukemia. N Engl J Med. 2016;374(12):11971198.

13. Cicconi L, Platzbecker U, Avvisati G, et al. Long-term results of alltrans retinoic acid and arsenic trioxide in non-high-risk acute promyelocytic leukemia: update of the APL0406 Italian-German randomized trial. Leukemia. 2020;34(3):914-918.

14. NAACCR Latino Research Work Group. NAACCR Guideline for Enhancing Hispanic-Latino Identification: Revised NAACCR Hispanic/Latino Identification Algorithm [NHIA v2]. Springfield (IL). North American Association of Central Cancer Registries, September 2005.

15. NAACCR Race and Ethnicity Work Group. NAACCR Asian Pacific Islander Identification Algorithm [NAPIIA v1.2.1]. Springfield (IL), August 2011 